\title{
Clinical, virological and immunological responses in Danish HIV patients receiving raltegravir as part of a salvage regimen
}

This article was published in the following Dove Press journal:

Clinical Epidemiology

25 May 2010

Number of times this article has been viewed

\author{
Frederik N Engsig' \\ Jan Gerstoft' \\ Gitte Kronborg ${ }^{2}$ \\ Carsten S Larsen ${ }^{3}$ \\ Gitte Pedersen ${ }^{4}$ \\ Anne MAudelin ${ }^{5}$ \\ Louise B Jørgensen ${ }^{5}$ \\ Niels Obel' \\ 'Department of Infectious Diseases, \\ Copenhagen University Hospital, \\ Rigshospitalet, Denmark; ${ }^{2}$ Department \\ of Infectious Diseases, Copenhagen \\ University Hospital, Hvidovre, \\ Denmark; ${ }^{3}$ Department of Infectious \\ Diseases, Aarhus University Hospital, \\ Aarhus, Denmark; ${ }^{4}$ Department \\ of Infectious Diseases, Aalborg \\ University Hospital, Aalborg, \\ Denmark; ${ }^{5}$ Department of Virology, \\ Statens Serum Institute, Copenhagen, \\ Denmark
}

Correspondence: Frederik Neess Engsig Department of Infectious Diseases, Rigshospitalet, Blegdamsvej 9, DK2 100 Copenhagen $\varnothing$, Denmark

Tel +4535457726

Fax +45 35456648

Email fren74@gmail.com
Background: Raltegravir is the first integrase inhibitor approved for treatment of HIV-infected patients harboring multiresistant viruses.

Methods: From a Danish population-based nationwide cohort of HIV patients we identified the individuals who initiated a salvage regimen including raltegravir and a matched cohort of HIV-infected patients initiating HAART for the first time. We compared these two cohorts for virological suppression, gain in CD4 count, and time to first change of initial regimen.

Results: We identified 32 raltegravir patients and 64 HIV patients who initiated HAART for the first time in the period 1 January 2006 to 1 July 2009. The virological and immunological responses in the raltegravir patients were comparable to those seen in the control cohort. No patients in the two cohorts died and no patients terminated raltegravir treatment in the observation period. Time to first change of initial regimen was considerably shorter for HAART-naïve patients.

Conclusion: We conclude that salvage regimens including raltegravir have high effectiveness in the everyday clinical setting. The effectiveness of the regimens is comparable to that observed for patients initiating HAART for the first time. The risk of change in the salvage regimens after initiation of raltegravir is low.

Keywords: HIV, raltegravir, salvage regime, efficacy, matched cohort

\section{Introduction}

Highly active antiretroviral therapy (HAART) was introduced more than a decade ago and the therapy has decreased mortality and morbidity of HIV patients dramatically. ${ }^{1,2}$ The first HAART regimens were composed of nucleoside reverse transcriptase inhibitors (NRTIs), non-nucleoside reverse transcriptase inhibitors (NNRTIs), and protease inhibitors (PIs). Especially the early regimens carried a substantial risk of failure and subsequent development of resistance to the three drug classes. ${ }^{3}$ Thus there has been a need for development of new drugs with activity against viruses resistant to the classical HAART regimens either as new drugs from the old classes without (or with limited) cross resistance to the older compounds or drugs from new classes with new antiretroviral mechanisms. The optimal choice for salvage therapy for HIV-infected patients has been shown to require at least 2 , and preferably 3 , fully active drugs. ${ }^{4-6}$ Until recently, salvage regimens used to treat patients harboring multidrug-resistant HIV generally included only one new agent from the classic drug classes added to an optimized background therapy which did not contain any fully active agents. This approach, conditioned by limited drug options, put patients at high risk of virological failure and resistance to the new agent, as well as to other agents in the same drug classes. ${ }^{7-9}$ A breakthrough has been the recent development of integrase inhibitors, 
which is a new class of antiretroviral drugs. ${ }^{10}$ One of these drugs - raltegravir - has demonstrated its activity in patients with virological failure on classical antiretroviral drugs. ${ }^{11}$ In the BENCHMARK randomized clinical trials, which were conducted in HIV-infected patients with limited treatment options, $62 \%$ of patients taking raltegravir plus optimized background treatment achieved plasma HIV RNA levels $<50$ copies/mL at week $48 .{ }^{11}$ Although the drug - often used together with other new drugs - has been proved effective in clinical trials and recently in 'real life' clinical settings, ${ }^{12-14}$ the long-term efficacy is not described and the effect compared to treatment in HAART-naïve patients remains to be established. In a nationwide cohort of HIV-infected patients, we identified the patients, who initiated raltegravir due to virological failure and a matched control cohort of patients initiating HAART for the first time. We compared these two cohorts for virological suppression, gain in CD4 cell count, and time to first change of initial regimen.

\section{Method}

\section{Setting}

Denmark had a population of 5.5 million as of 31 December 2008, with an estimated HIV prevalence of approximately $0.07 \%$ in the adult population. ${ }^{15,16}$ Patients with HIV infection are treated in one of the country's 8 specialized medical centers, where they are seen on an outpatient basis at intended intervals of 12 weeks. Antiretroviral treatment is provided free of charge to all HIV-infected residents of Denmark.

\section{Data sources}

The Danish HIV Cohort study (DHCS) is a populationbased prospective nationwide cohort study of all HIVinfected individuals 16 years or older at diagnosis and who are treated at Danish HIV centers after 1 January $1995 .{ }^{17}$ Patients are consecutively enrolled, and multiple registrations are avoided through the use of a unique 10-digit civil registration number assigned to all individuals in Denmark at birth or upon immigration. Data are updated yearly and include demographics, date of HIV infection, AIDSdefining events, date and cause of death, and antiretroviral treatment. The national criteria for initiating HAART have been described previously. ${ }^{18} \mathrm{CD} 4$ cell counts and HIV-RNA measurements are extracted electronically from laboratory data files. On 31 December 2008 the cohort included 5206 Danish HIV-infected residents.

Sequences containing the reverse transcriptase and the protease gene (1300 base pairs) were obtained through population-based sequencing using ViroseqTM HIV-1 genotyping System v.2 (Abbott Diagnostics, Foster City, USA) and collected from the Danish HIV Sequence Database. ${ }^{19}$ All sequences were obtained from plasma samples collected before start of raltegravir. Resistance mutations against all the approved NRTI, NNRTI, and PI drugs were identified and classified using the Stanford Genotypic Resistance Interpretation Algorithm (version 6.0.7; HIV Drug Resistance Database, Stanford, CA, USA). ${ }^{20}$

\section{Study populations}

Raltegravir cohort patients: From DHCS we included all HIV-1 positive patients, who 1) started raltegravir after 1 January 2006 and before 1 July 2009, 2) had been treated with HAART previously, 3) had at least 2 viral load (VL) tests done prior to initiation of raltegravir treatment, 4) had virological failure prior to start of raltegravir and, 5) did not participate in randomized clinical trials on raltegravir. Virological failure was defined as VL $>500$ copies $/ \mathrm{mL}$ in the two latest VL tests prior to raltegravir initiation while on HAART treatment.

Control cohort patients: From DHCS we identified a control cohort of HIV-infected patients who started HAART for the first time after 1 January 2006 and before 1 July 2009. From this population we extracted 2 control patients for each raltegravir patient, each matched by gender, race (Caucasian, Black, and other), route of HIV infection (homosexual, heterosexual, injection drug user [IDU], and other) and age (intervals of $<20$ years, 20 to 30 years, 30 to 40 years, $>50$ years). Due to lack of controls, matching with younger patients was allowed for 1 raltegravir patient of African origin, with homosexual HIV transmission, and older than 50 years.

\section{Statistics}

Demographics and characteristics were computed for the raltegravir and control cohort, as were characteristics of treatment regimens used prior to raltegravir initiation and with raltegravir.

Index date was defined as date of first initiation of raltegravir or of HAART in the raltegravir and control cohort, respectively. We used Kaplan-Meier analysis to describe time to first change in antiretroviral regime and in these analysis, time was calculated from index date to date of last outpatient visit, 1 July 2009 or date of first change in antiretroviral regime, whichever came first. In other analysis time was calculated from index date to 1 July 2009, date of immigration or date of death, whichever came first. 
Using a previously described method we grouped all CD4 cell measurements and VL tests in the observation period in 12-week intervals and calculated the proportion of VL tests $<51$ copies $/ \mathrm{mL}$ and the median CD4 cell count from 96 weeks before to 72 weeks after index date. ${ }^{17}$ Differences in median CD4 cell count between the two cohorts at index date and after 72 weeks were evaluated by Fisher's exact test. We evaluated effect on CD4 cell count from baseline to week 48 and 72 .

The study was approved by the Danish Data Protection Agency. The study is registered with ClinicalTrials.gov (Identifier: NCT01061957). SPSS statistical software, Version 15.0 (Norusis; SPSS Inc, Chicago, Illinois, USA) and R software, version 2.8.1, was used for data analysis.

\section{Results}

From DHCS we identified 32 patients who started a salvage regimen including raltegravir and a control cohort of 64 patients naïve to antiretroviral drugs who started HAART with 50.2 and 142.8 years of observation, respectively. No raltegravir or control patients were lost to follow up. As seen from Table 1 the raltegravir patients and the control patients were well matched with respect to age, gender, race and route of HIV infection, but due to the differences in inclusion criteria differed in time from HIV diagnosis to index date, AIDS-defining events prior to index date, and VL and $\mathrm{CD} 4$ cell count at index date. The raltegravir cohort had been treated with several different antiretroviral regimens and a major fraction was on a regimen including a boosted protease inhibitor prior to the start of raltegravir (Table 2). Ten (31.3\%) of the raltegravir patients were treated with zidovudin prior to start of raltegravir compared with $3(9.4 \%)$ after start of raltegravir (Appendix 1).

All raltegravir patients had primary resistance mutations against 1 or more drug classes, $32(100 \%)$ had primary resistance mutations against 2 or more drug classes and $26(81.3 \%)$ had primary resistance mutations against 3 classes. NRTI resistance mutations were found in 32 $(100 \%)$ raltegravir patients, 29 (90.1\%) had NNRTI resistance mutations, and $29(90.1 \%)$ had primary PI resistance mutations.

The optimized background salvage regimens started at the time of raltegravir initiation included treatment with etravirine in $30 \%$ of the raltegravir patients and darunavir in almost $90 \%$ of the patients ( $84.4 \%$ initiated boosted darunavir at the same time as raltegravir) (Table 3). Two (6.3\%) of the patients did not receive any NRTI.

No raltegravir or control patients died in the observation period and an AIDS-defining event occurred in $1(3.1 \%)$ and $5(7.8 \%)$ patients in the raltegravir cohort and control cohort, respectively. After the initiation of raltegravir or HAART, the majority of the two cohorts obtained a VL $<50$ copies $/ \mathrm{mL}$ after 72 weeks of treatment (Figure 1). All raltegravir patients achieved virological suppression in the observation period. Notably the

Table I Characteristics and demographics of patients treated with raltegravir and a control population naïve to HAART

\begin{tabular}{|c|c|c|}
\hline Characteristics & Raltegravir patients $\mathbf{N}=32$ & Controls N $=64$ \\
\hline Male gender & $21(65.6 \%)$ & $42(65.6 \%)$ \\
\hline Age at time of index date, median (IQR), years & $50.8(42.5-55.1)$ & $47.3(40.5-56.3)$ \\
\hline \multicolumn{3}{|l|}{ Race } \\
\hline Caucasian & $23(71.9 \%)$ & 46 (71.9\%) \\
\hline Black & $7(21.9 \%)$ & $14(2 \mid .9 \%)$ \\
\hline Other & $2(6.2 \%)$ & $4(6.2 \%)$ \\
\hline \multicolumn{3}{|l|}{ Route of HIV infection } \\
\hline Men who have sex with men & $15(46.9 \%)$ & $30(46.9 \%)$ \\
\hline Heterosexually infected & $17(53.1 \%)$ & $34(53.1 \%)$ \\
\hline Injection drug user & None & None \\
\hline Other & None & None \\
\hline Diagnosed with HIV before I January 1995 & $9(28.1 \%)$ & $5(7.8 \%)$ \\
\hline CD4 cell count at index date, median (IQR), cells $/ \mu \mathrm{L}$ & $280(60-291)$ & $221(18 I-472)$ \\
\hline Viral load at index date, median (IQR), log 10 copies $/ \mathrm{mL}$ & $2.45(1.60-3.16)$ & $4.40(3.24-5.46)$ \\
\hline Time from HIV diagnosis to index date, median (IQR), years & I5.3 (|2.5-|8.8) & $0.2(0.1-3.4)$ \\
\hline Time from HAART start to index date, median (IQR), years & $10.7(10.0-11.3)$ & - \\
\hline One or more AIDS-defining events prior to index date & $14(43.8 \%)$ & II (I7.2\%) \\
\hline
\end{tabular}

Abbreviations: IQR, interquartile range. 
Table 2 Characteristics of HAART regimens used prior to first raltegravir regimen

\begin{tabular}{ll}
\hline $\begin{array}{l}\text { Number of all NRTIs, NNRTIs, and PIs used } \\
\text { prior to first raltegravir regimen }\end{array}$ & Median (IQR) \\
\hline NRTI & $5(5-7)$ \\
NNRTI & I (I-2) \\
PI & $4(4-5)$ \\
\hline Drugs included in the last HAART regimen & N (\%) \\
prior to first raltegravir initiation & \\
\hline NRTI & 3 I (96.9) \\
I NRTI & $2(6.3)$ \\
2 NRTI & $22(68.8)$ \\
3 NRTI & $7(21.9)$ \\
NNRTI & $4(12.5)$ \\
PI & $32(100)$ \\
Boosted PI & $28(87.5)$ \\
$\quad$ Non-boosted PI & $4(12.5)$ \\
\hline
\end{tabular}

Abbreviations: IQR, interquartile range; NRTIs, nucleoside reverse transcriptase inhibitors; NNRTIs, non-nucleoside reverse transcriptase inhibitors; Pls, protease inhibitors.

virological response was almost equivalent in the raltegravir and the control cohorts at week 72 (100\% vs $83.3 \%$, respectively).

The raltegravir patients had a higher median CD4 cell count at index date than the control cohort $(280$ cells $/ \mu \mathrm{L}$ [IQR, interquartile range; 60-291] vs 221 cells/ $\mu \mathrm{L}$ [IQR; 181-472], $P=0.02$ ), (Figure 2). Both cohorts had a strong CD4 response to raltegravir and HAART initiation, respectively. The median CD4 cell count increase from index date to week 48 was 180 cells/ $\mu \mathrm{L}$ (IQR; 40-244) in the raltegravir cohort and 160 cells/ $\mu \mathrm{L}$ (IQR; $81-239$ ) in the control cohort. After 72 weeks of treatment the raltegravir cohort continued to have a higher CD4 cell count compared with the control patients (558 cells/ $\mu \mathrm{L}$ [IQR; 420-656]

Table 3 Antiretroviral drugs in first raltegravir regimen

\begin{tabular}{ll}
\hline Antiretroviral drugs & $\mathbf{N}(\%)$ \\
\hline NRTI & $24(75.0)$ \\
I NRTI & $3(9.4)$ \\
$2 \mathrm{NRTI}$ & $17(53.1)$ \\
$3 \mathrm{NRTI}$ & $4(12.5)$ \\
NNRTI & $14(43.8)$ \\
Etravirine & $12(37.5)$ \\
Boosted PI & $30(93.8)$ \\
Darunavir & $28(87.5)$ \\
Non-boosted PI & $2(6.7)$ \\
CCR5 inhibitor (maraviroc) & $3(9.4)$ \\
Fusion inhibitor (enfuvirtide) & $\mathrm{I}(3.1)$ \\
\hline
\end{tabular}

Abbreviations: NRTIs, nucleoside reverse transcriptase inhibitors; NNRTIs, nonnucleoside reverse transcriptase inhibitors; Pls, protease inhibitors.

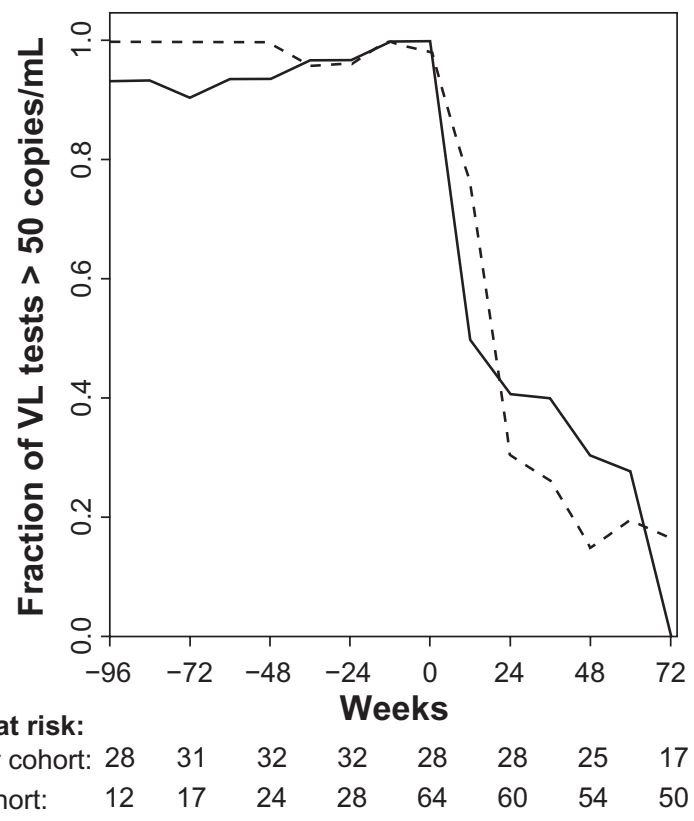

Figure I Fraction of viral load (VL) tests below $5 \mathrm{I}$ copies $/ \mathrm{mL}$ in 12 weeks intervals for the raltegravir cohort (full line) and the control cohort (broken line). Week 0 is start of raltegravir or HAART (index date).

vs 399 cells/ $\mu \mathrm{L}$ [IQR; 286-529], $P=0.01$ ). The increase in median CD4 cell count from baseline to week 72 was 270 cells/ $\mu \mathrm{L}$ (IQR; 146-390) in the raltegravir cohort and 183 cells $/ \mu \mathrm{L}$ (IQR; 98-265) in the control cohort.

None of the raltegravir patients stopped raltegravir in the study period. Figure 3 illustrates time to first change in the HAART regimens in the two cohorts. Almost two thirds of the raltegravir patients were on the initial salvage regimen after 1 year compared with less than $50 \%$ of the patients in the control cohort.

\section{Discussion}

In an observational, nationwide and population-based study we found a high effectiveness of salvage regimens including raltegravir. The clinical progression, immunological and virological responses were comparable to those seen in a matched cohort of HIV-infected patients initiating HAART for the first time. The major strengths of the study are its nationwide population-based design, combined with complete follow-up.

The impact of the raltegravir regimens on VL was comparable to that seen in patients initiating HAART for the first time. Compared to other studies we observed a slightly lower initial virological response in the raltegravir patients, ${ }^{12,14}$ but after 72 weeks all patients were virologically suppressed. As almost all of the patients initiated boosted darunavir as part of salvage regimens, we were not able 


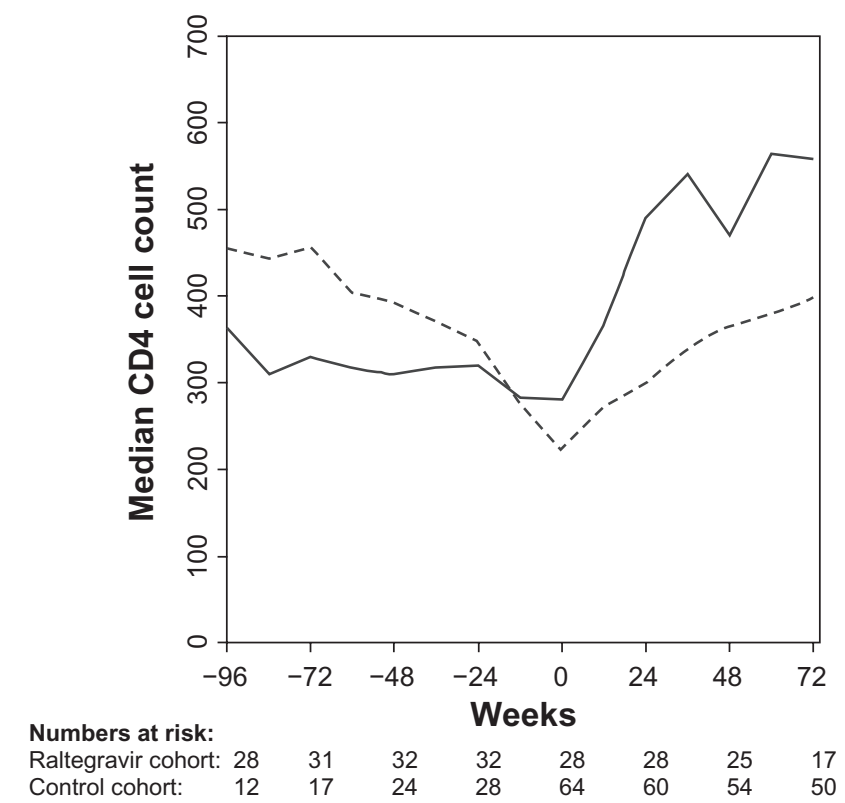

Figure 2 Median CD4 cell count at I2-week intervals for the raltegravir cohort (full line) and the control cohort (broken line). Week 0 is start of raltegravir or HAART (index date).

to distinguish between the raltegravir and the darunavir effects. The strong antiretroviral effect of darunavir has been shown in a study by Arribas et al who demonstrated that the antiretroviral effect of monotherapy with boosted darunavir was comparable to that obtained with triple combination therapy. ${ }^{21}$ Further, the SWITCHMRK study, in which raltegravir underperformed compared to boosted lopinavir in patients with regimens primarily dependent on these two

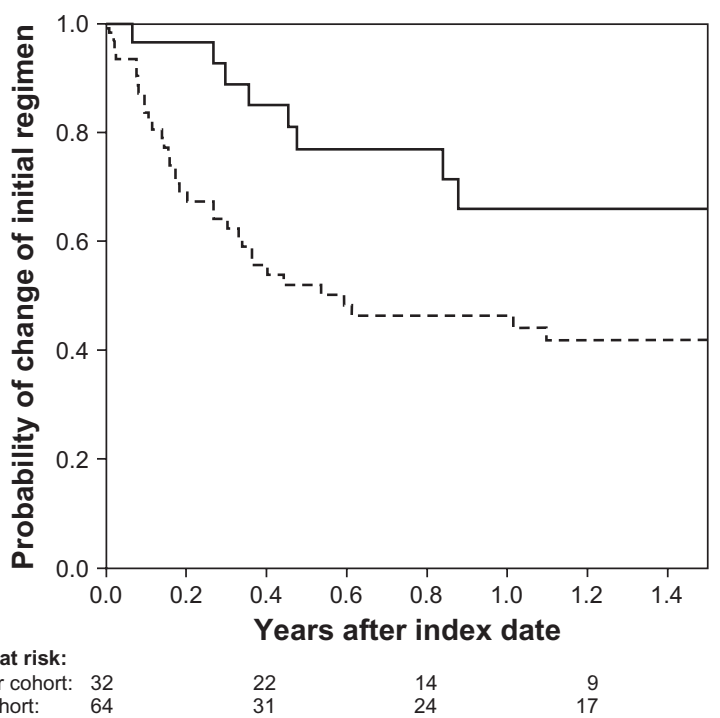

Figure 3 Kaplan-Meir curve of time to change in first raltegravir-containing (full line) or HAART (broken line) regimens. drugs, indicates the importance of an optimized background regimen including a boosted protease inhibitor. ${ }^{11,22}$ In the present study only one third of the patients used etravirine as this drug was not available in Denmark until the last half of the study period.

Surprisingly, the initial CD4 cell recovery in the raltegravir cohort was higher than that of the controls and that found in the TRIO trial. ${ }^{12}$ At week 72 the raltegravir cohort exceeded the control cohort, rising to a median CD4 cell count above 500 cells $/ \mu \mathrm{L}$. A similar increase was observed in perinatally infected adolescents. ${ }^{23}$ If a previous drop in the CD4 count had induced the change to the raltegravir regimens, regression towards the mean could partly explain a subsequent increase in CD4 count. However, the course of the CD4 cell count in the observation period prior to introduction of the salvage regimen does not suggest such a phenomenon. It may be hypothesized that patients surviving despite years of virological failure may have a greater CD4 generating capacity and the increase in CD4 count therefore may be a healthy survivor phenomenon. The discontinuation of zidovudine in a large proportion of the patients prior to salvage therapy could partly explain this unprecedented large CD4 increase.

Time to change of the initial raltegravir regimen was considerably longer than time to change in initial HAART regimen in the control cohort, thus supporting the findings of Willig et al. ${ }^{24}$ We, however, compared regimens given in two very different situations. The salvage patients were facing potential progression of the underlying infection due to limited treatment options, thus the patients and their healthcare providers presumably accepted far greater side effects before changing regimens compared to the HAART-naïve patients. Still, our results indicate a high tolerance of raltegravir.

The mortality in HIV patients on HAART is low and may approach that of the background cohort. ${ }^{1}$ In accordance with this we observed no death in the two cohorts and no increased risk of AIDS-defining illness in the raltegravir group. However, in order to evaluate clinical endpoints a substantially larger study population and longer observation period are needed.

We conclude that salvage regimens including raltegravir are effective not only in clinical trials, but also have high effectiveness in the everyday clinical setting. The effectiveness of the regimens is comparable to that observed for patients initiating HAART for the first time. The risk that raltegravir is stopped prematurely or the salvage regimens are changed after initiation is low. 


\section{Acknowledgments}

We thank the staff of our clinical departments for their continuous support and enthusiasm. Centres in The Danish HIV Cohort Study: Departments of Infectious Diseases at Copenhagen University Hospitals, Rigshospitalet (J Gerstoft, N Obel,) and Hvidovre (G Kronborg), Odense University Hospital (B Røge), Aarhus University Hospitals, Skejby (CS Larsen) and Aalborg (G Pedersen), Herning Hospital (AL Laursen), Helsingør Hospital (L Nielsen), and Kolding Hospital (J Jensen).

\section{Disclosures}

Supported in part by a research grant (grant number 37593) from the Investigator-Initiated Studies Program of MSD, a subsidiary of Merck and Co, Inc. The opinions expressed in this paper are those of the authors and do no necessarily represent those of MSD, a subsidiary of Merck and Co, Inc. The funders had no role in the study design; in the collection, management, analysis, and interpretation of data; in the preparation, review, or approval of the manuscript; or in the decision to submit the article for publication. The researchers are independent from the funders.

N Obel has received research funding from Roche, Bristol-Myers Squibb, Merck Sharp \& Dohme, GlaxoSmithKline, Abbott, Boehringer Ingelheim, Janssen-Cilag, and Swedish Orphan. F Engsig has received research funding from Merck Sharp \& Dohme. J Gerstoft has received research funding from Abbott, Roche, Bristol-Myers Squibb, Merck Sharp \& Dohme, Pharmacia, GlaxoSmithKline, Swedish Orphan, and Boehringer Ingelheim.

\section{References}

1. Lohse N, Hansen AB, Pedersen G, et al. Survival of persons with and without HIV infection in Denmark, 1995-2005. Ann Intern Med. 2007;146(2):87-95.

2. Engsig FN, Hansen AB, Gerstoft J, Kronborg G, Larsen CS, Obel N. Inpatient admissions and outpatient visits in persons with and without HIV infection in Denmark, 1995-2007. AIDS. 2010;24(3):457-461.

3. Lohse N, Obel N, Kronborg G, et al. Declining risk of triple-class antiretroviral drug failure in Danish HIV-infected individuals. AIDS. 2005;19(8):815-822.

4. Department of Health and Human Services. Guidelines for the use of antiretroviral agents in HIV-1-infected adults and adolescent [online]. Available from: http://www.aidsinfo nih gov/ContentFiles/ AdultandAdolescentGL pdf. Accessed 2010 January 1.

5. Deeks SG. Treatment of antiretroviral-drug-resistant HIV-1 infection. Lancet. 2003;362(9400):2002-2011.

6. Ledergerber B, Lundgren JD, Walker AS, et al. Predictors of trend in CD4-positive T-cell count and mortality among HIV-1-infected individuals with virological failure to all three antiretroviral-drug classes. Lancet. 2004;364(9428):51-62.
7. Gulick RM, Hu XJ, Fiscus SA, et al. Randomized study of saquinavir with ritonavir or nelfinavir together with delavirdine, adefovir, or both in human immunodeficiency virus-infected adults with virologic failure on indinavir: AIDS Clinical Trials Group Study 359. J Infect Dis. 2000;182(5):1375-1384.

8. Hammer SM, Vaida F, Bennett KK, et al. Dual vs single protease inhibitor therapy following antiretroviral treatment failure: a randomized trial. JAMA. 2002;288(2):169-180.

9. Lederman MM, Miller V, Weller I, Deeks SG. A new approach for 'deep salvage' trials in advanced HIV infection. AIDS. 2007;21(12): 1503-1506.

10. Hazuda DJ, Felock P, Witmer M, et al. Inhibitors of strand transfer that prevent integration and inhibit HIV-1 replication in cells. Science. 2000;287(5453):646-650.

11. Steigbigel RT, Cooper DA, Kumar PN, et al. Raltegravir with optimized background therapy for resistant HIV-1 infection. $N$ Engl J Med. 2008;359(4):339-354.

12. Yazdanpanah Y, Fagard C, Descamps D, et al. High rate of virologic suppression with raltegravir plus etravirine and darunavir/ritonavir among treatment-experienced patients infected with multidrugresistant HIV: results of the ANRS 139 TRIO trial. Clin Infect Dis. 2009;49(9):1441-1449.

13. Grant PM, Palmer S, Bendavid E, et al. Switch from enfuvirtide to raltegravir in virologically suppressed HIV-1 infected patients: effects on level of residual viremia and quality of life. J Clin Virol. 2009;46(4):305-308.

14. Scherrer AU, von Wyl V, Fux CA, et al. Implementation of raltegravir in routine clinical practice: selection criteria for choosing this drug, virologic response rates, and characteristics of failures. J Acquir Immune Defic Syndr. 2010;53(4):464-471.

15. Statistics Denmark [online]. Available from: http://www.sdt.dk. Accessed 2010 January 1.

16. Lohse N, Hansen AB, Jensen-Fangel S, et al. Demographics of HIV-1 infection in Denmark: results from the Danish HIV Cohort Study. Scand J Infect Dis. 2005;37(5):338-343.

17. Jensen-Fangel S, Pedersen L, Pedersen C, et al. The effect of race/ ethnicity on the outcome of highly active antiretroviral therapy for human immunodeficiency virus type 1-infected patients. Clin Infect Dis. 2002;35(12):1541-1548.

18. Obel N, Engsig FN, Rasmussen LD, Larsen MV, Omland LH, Sorensen HT. Cohort profile: the Danish HIV cohort study. Int J Epidemiol. 2009;38(5):1202-1206.

19. Audelin AM, Lohse N, Obel N, Gerstoft J, Jorgensen LB. The incidence rate of HIV type-1 drug resistance in patients on antiretroviral therapy: a nationwide population-based Danish cohort study 1999-2005. Antivir Ther. 2009;14(7):995-1000.

20. Liu TF, Shafer RW. Web resources for HIV type 1 genotypic-resistance test interpretation. Clin Infect Dis. 2006;42(11):1608-1618.

21. Arribas JR, Horban A, Gerstoft J, et al. The MONET trial: darunavir/ ritonavir with or without nucleoside analogues, for patients with HIV RNA below 50 copies/ml. AIDS. 2010;24(2):223-230.

22. Cocohoba J. The SWITCHMRK studies: substitution of lopinavir/ ritonavir with raltegravir in HIV-positive individuals. Expert Rev Anti Infect Ther. 2009;7(10):1159-1163.

23. Thuret I, Chaix ML, Tamalet C, et al. Raltegravir, etravirine and r-darunavir combination in adolescents with multidrug-resistant virus. AIDS. 2009;23(17):2364-2366.

24. Willig JH, Aban I, Nevin CR, et al. Comparative regimen durability and discontinuation reasons among 3-class experienced patients in a US clinical cohort [poster]. EACs. 2009 Nov 11-14; Cologne, Germany. 
Appendix I HAART regimen in the raltegravir cohort prior to and after start of raltegravir

\begin{tabular}{|c|c|c|}
\hline Patient & Pre-raltegravir treatment & On-raltegravir treatment \\
\hline \#I & ZDV, 3CT, ABC, RTV, DRV & ZDV, 3CT,ABC, RTV, DRV, RAL \\
\hline$\# 2$ & ZDV, 3CT, RTV & FTC,TDF, DRV, NVP, ETV, RAL \\
\hline \#3 & ZDV, 3CT, RTV & 3CT, ABC, RTV, DRV, ETV, RAL \\
\hline$\# 4$ & TDF, RTV, EFV & RTV,DRV, ETV, RAL \\
\hline$\# 5$ & ZDV,TDF,ATV & FTC,TDF,ATV, RAL \\
\hline \#6 & 3CT,ABC,TDF, RTV,ATV & RTV, DRV, ETV, RAL \\
\hline \#7 & 3CT,ABC, RTV,ATV, EFV & FTC,TDF, RTV, DRV, RAL \\
\hline$\# 8$ & 3CT,ABC, RTV,ATV & FTC,TDF, RTV, DRV, RAL \\
\hline \#9 & RTV, LPV, EFV & FTC,TDF, RAL \\
\hline \#10 & $3 C T, A B C, R T V, T P V, t 20$ & RTV, DRV, RAL, MVC \\
\hline \#II & ZDV, 3CT,TDF, RTV,TPV & FTC,TDF, RTV, DRV, ETV, RAL \\
\hline$\# 12$ & 3CT,ABC, RTV,ATV & RTV, DRV, ETV, RAL \\
\hline$\# 13$ & ZDV, 3CT,TDF, RTV,TPV & FTC,TDF, RTV, DRV, RAL \\
\hline \#14 & ZDV, 3CT,ATV & ABC,TDF, DRV, RAL \\
\hline \#15 & D4T,ABC,TDF, SQV, RTV & RTV, DRV, ETV, RAL \\
\hline$\# 16$ & 3CT, DDI,TDF, RTV,TPV & FTC, DDI,TDF, RTV, DRV, RAL,T20 \\
\hline \#17 & 3CT,ABC, RTV & TDF, RTV, RAL \\
\hline$\# 18$ & DDI,TDF, RTV,AMP & RTV, DRV, RAL, ETV \\
\hline \#19 & 3CT, D4T, DDI, RTV, IDV, & ZDV,3CT, ABC, RTV, ETV, RAL \\
\hline \#20 & DDI,ABC, RTV & RTV, DRV, RAL \\
\hline \#21 & 3CT, D4T, RTV & 3CT, DDI, RTV, DRV, RAL \\
\hline \#22 & 3CT, D4T, RTV & FTC,TDF, DRV, ETV, RAL \\
\hline \#23 & DDI,ABC, RTV & DRV, EFV, ETV, RAL \\
\hline \#24 & DDI,ABC,ATV & FTC,TDF, RTV, DRV, RAL \\
\hline \#25 & FTC,TDF, RTV & FTC, RTV, DRV, RAL \\
\hline \#26 & DDI,ABC, RTV,ATV & FTC,TDF, RTV, DRV, RAL \\
\hline$\# 27$ & ABC, RTV & 3CT, ABC, RTV, DRV, RAL \\
\hline \#28 & ZDV,TDF, SQV, RTV & ZDV, FTC,TDF, SQV, RTV, DRV, RAL, MVC \\
\hline \#29 & ABC, TDF, RTV, & ABC, TDF, RTV, DRV, RAL \\
\hline \#30 & ABC,TDF, RTV,TPV & 3CT, ABC, RTV, DRV, ETV, RAL \\
\hline \#31 & ZDV, 3CT, RTV, EFV & FTC,TDF, DRV, RAL, MVC \\
\hline \#32 & ZDV, DDI, RTV, IDV & TDF, RTV, DRV, NVP, RAL \\
\hline
\end{tabular}

Abbreviations:TDF, tenofovir; RTV, ritonavir;SQV, saquinavir; FTC, emtricitabine; DRV, darunavir; DDI, didanosine; ABC, abacavir; 3TC, lamivudine; D4T, stavudine; LPV, lopinavir; EFV, efavirenz;TPV, tipranavir; FPV, fosamprenavir; NVP, nevirapine; ATV, atazanavir; IDV, crixivan; ZDV, zidovudine; ETV, etravirine; MVC, maraviroc; AMP, amprenavir.

\section{Publish your work in this journal}

Clinical Epidemiology is an international, peer-reviewed, open access journal focusing on disease and drug epidemiology, identification of risk factors and screening procedures to develop optimal preventative initiatives and programs. Specific topics include: diagnosis, prognosis, treatment, screening, prevention, risk factor modification, systematic

Submit your manuscript here: http://www.dovepress.com/clinical-epidemiology-journa

\section{Dovepress}

reviews, risk \& safety of medical interventions, epidemiology \& biostatical methods, evaluation of guidelines, translational medicine, health policies \& economic evaluations. The manuscript management system is completely online and includes a very quick and fair peer-review system, which is all easy to use. 